EESTI NSV TEADUSTE AKADEEMIA TOIMETISED. IX KÖIDE

FOOSIKALIS-MATEMAATILISTE JA TEHNILISTE TEADUSTE SEERIA, 1960, NR. 3

ИЗВЕСТИЯ АКАДЕМИИ НАУК ЭСТОНСКОН ССР. ТОМ IX СЕРИЯ ФИЗИКО-МАТЕМАТИЧЕСКИХ И ТЕХНИЧЕСКИХ НАУК. 1960, № 3

\title{
МЕТОДЫ СУММИРОВАНИЯ, СОХРАНЯЮЩИЕ СХОДИМОСТЬ
}

\section{Э. Юримяэ,}

\author{
кандидат физико-математичеоких наук
}

В настоящей статье рассматриваются обобщенные методы суммирования, определенные С и ко рским $\left[{ }^{2}\right]$. Для этих методов обобщается понятие о сохранении сходимости, и при помощи последнего изучаются некоторые общие свойства методов Сикорского, сохраняющих сходимость. Так как матричные, непрерывные функциональные и интегральные методы суммирования являются частными случаями метода Сикорского, то из общей теории $(\$ \S 2-4)$ получаются как следствия известные уже результаты о матричных и непрерывных методах $(\$ 5)$, а также новые результаты об интегральных методах (\$7).

\section{§ 1. Определение метода}

В настоящем параграфе рассматривается определение метода суммирования, данное в работе [2]. Из него как частные случаи получаем матричные методы (для числовых последовательностей), функциональные методы (см. [3,12]) и инте́гральные методы (см. [9]).

Пусть $N_{0}$ и $\Re_{0}$ - некоторые компактные множества действительных чисел, $n_{0}$ и $v_{0}$ соответственно их точки прикосновения (т. е. неизолированные точки). Обозначим

$$
\begin{aligned}
& N=N_{0}-\left\{n_{0}\right\}, \\
& \Re=\Re_{0}-\left\{v_{0}\right\} .
\end{aligned}
$$

Предположим еще, что $N_{0}$ является метрическим пространством, в котором будем рассматривать множества

$$
N_{i \mu}=\underset{n \in N}{E}\left(\rho\left(n, n_{0}\right) \geqslant \frac{1}{\mu}\right) \quad(\mu=1,2, \ldots)
$$

Пусть $X$ - фиксированное $F$-пространство (т. е. полное метрическое локально выпуклое пространство), в котором каждому числу $v \in \Re$ соответствует некоторое линейное преобразование $T$, пространства $X$ в себя таким образом, что (вместо $T_{v}(x)$ пишем $x_{v}$ )

(a) $\lim _{v \rightarrow v_{0}} x_{v}=x \quad$ в $X$;

(b) $T_{v} T_{v^{\prime}}=T_{v} T_{v}=T_{\min \left(v, v^{\prime}\right)}\left(\right.$ т. e. $\left.\left(x_{v}\right)_{v^{\prime}}=x_{\min \left(v, v^{\prime}\right)}\right)$.

Предположим также, что каждому числу $n \in N$ соответствует линейный, не обязательно непрерывный функционал $f_{n}$, определенный на 
линейном пространстве $X^{\prime}$ всех элементов $x$, (где $x \in X$ и $v \in \Re$ ) таким образом, что выполнены условия

(c) для каждого фиксированного $x \in X$ функция $f_{n v}(x)=f_{n}\left(x_{v}\right)$ переменных $n, v$ непрерывна на $N \times \Re$;

(d) при фиксированных $n \in N$ и $v \in \Re$ линейный функционал $f_{n v}$ (определенный на $X$ ) непрерывен.

Всякая система

$$
\mathfrak{A}=\left[X, \Re, v_{0}, N, n_{0},\left\{T_{v}\right\},\left\{f_{n}\right\}\right],
$$

удовлетворяющая вышеуказанным условиям, называется методом суммирования. В дальнейшем, изучая свойства таких методов суммирования, будем предполагать, что все вышеуказанные условия выполнены. В случае метода $\mathfrak{A} \mathfrak{V}$-суммой элемента $x$ называется конечный предел

$$
\lim _{n \rightarrow n_{0} v \rightarrow v_{0}} \lim _{n}\left(x_{v}\right)=f(x) .
$$

Сикорский [2] показал, что множество $X^{\circ}$ всех элементов $X$, таких, что $f_{n}\left(x_{v}\right)$ сходится почти равномерно на $N$ (т. е. равномерно на всяком компактном подмножестве множества $N$ ), при $v \rightarrow v_{0}$ является $F$-пространством с топологией, определенной всеми квазинормами из $X$ и квазинормами

$$
\|x\|_{\mu-}=\sup _{\substack{\nu \in \Re_{i} \\ n \in N_{\mu}}}\left|f_{n}\left(x_{\nu}\right)\right| \quad(\mu=1,2, \ldots)
$$

Полем суммируемости метода $\mathfrak{A}$ называется линейное пространство $\mathfrak{P} *$ всех $x \in X^{\circ}$, таких, что существует конечный предел

$$
\lim _{n \rightarrow n_{0}} f_{n}(x)=f(x) \text {. }
$$

Полем суммируемости $\mathfrak{A} *$ является $F$-пространством со всеми квазинормами из $X^{\circ}$ и с квазинормой

$$
\|x\|_{0}=\sup _{n \in N}\left|f_{n}(x)\right| .
$$

Сикорский $\left[{ }^{2}\right]$ показал также, что всякий линейный непрерывный функционал $\varphi$ на $\mathfrak{2}^{*}$ имеет вид

$$
\varphi(x)=F(x)+\int_{N_{0}} f_{n}(x) d \tau(n),
$$

тде $F$ - линейный непрерывный функционал на $X^{0}$ и $\tau(n)-$ счетноаддитивная функция на классе всех борелевых подмножеств множества $N_{0}$.

Пусть $\overline{\mathfrak{A}}-$ множество всех $x \in \mathfrak{A}^{*}$, таких, что

$1^{0} f\left(x_{v}\right)=\lim _{n \rightarrow n_{0}} f_{n}\left(x_{v}\right)$ существует при всяком $v \in \Re$,

$2^{0} \lim _{v \rightarrow v_{0}} f\left(x_{v}\right)$ существует,

$3^{0} \sup _{\nu \in \Re}\left|f_{n}\left(x_{v}\right)\right|<\infty$.

$n \in N$ 
Из условий $1^{0}-3^{0}$ вытекает, что при $x \in \overline{\mathfrak{X}}$ каждый линейный непрерывный функционал $\varphi$ выражается формулой

$$
\varphi(x)=\lim _{v \rightarrow v_{0}} \varphi\left(x_{v}\right)+\tau\left(n_{0}\right)\left[f(x)-\lim _{v \rightarrow v_{0}} f\left(x_{v}\right)\right] .
$$

\section{§ 2. Корегулярные и конулевые методы}

В настоящем параграфе мы обобщаем понятия корегулярные и конулевые для методив

$$
\mathfrak{U}=\left[X, \mathfrak{R}, v_{0}, N, n_{0},\left\{T_{v}\right\},\left\{\tilde{f}_{n}\right\}\right],
$$

определенных в $\S 1$. Это обобщение основывается на определениях корегулярных и конулевых обобщенных матричных методов (см. $\left.{ }^{4}\right]$ ).

Пусть $X_{1}$ - такое замкнутое подпространство пространства $X$, что $X_{1} \supset X^{\prime}$ (см. стр. 257). Через пополнение $X^{\prime}$ образуем подпространство $X_{0}$ пространства $X_{1}$. Пусть $X_{0} \neq X_{1}$ (теоретико-множественно). Так мы получим пространства $X_{0}, X_{1}$ и $X$, для которых имеют место соотношения

$$
X_{0} \subset X_{1} \subset X \text {. }
$$

Благодаря последнему обстоятельству, из сходимости в $X_{0}$ вытекает сходимость в $X_{1}$, а из сходимости в $X_{1}-$ сходимость в $X$ (см. [ $\left.\left.{ }^{9}\right]\right)$.

В дальнейшем будем рассматривать методы $\mathfrak{A}$, при которых $\overline{\mathfrak{A}} \supset X_{1}$. Тогда $X_{0}, X_{1}, \mathfrak{U}^{*}$ и $X\left(X_{0} \subset X_{1} \subset \mathfrak{U}^{*} \subset X\right)$ образуют последовательность пространств, для которых из сходимости более узкого пространства вытекает сходимость более широкого пространства.

Мы называем метод $\mathfrak{A}=\left[X, \mathfrak{R}, v_{0}, N, n_{0}\left\{T_{v}\right\},\left\{f_{n}\right\}\right] \quad X_{1}-$ кор егу л я р ны м, если в пространстве $X_{1}$ существует элемент $x^{*}$, для которого $x_{v}^{*}$ не сходится слабо к $x^{*}$ в пространстве $\mathfrak{U}^{*}$. Из (1) получаем необходимое и достаточное условие для $X_{1}$-корегулярности метода $\mathfrak{A}$.

Т ео о м а 2.1. Метод $\mathfrak{2}$ является $X_{1}$-корегулярным тогда и только тогда, когда в $X_{1}$ найдется такой элемент $x^{*}$, при котором

$$
f\left(x^{*}\right) \neq \lim _{v \rightarrow v_{0}} f\left(x_{v}^{*}\right) .
$$

Все остальные методы, для которых $\overline{\mathfrak{U}} \supset X_{1}$, мы называем $X_{1}$-к о н у л е в ы м и.

Т ео рем а 2.2. Метод $\mathfrak{A}$ является $X_{1}$-конулевым тогда и только тогда, когда

$$
f(x)=\lim _{v \rightarrow v_{0}} f\left(x_{v}\right) \quad \text { при всех } x \in X_{1} .
$$

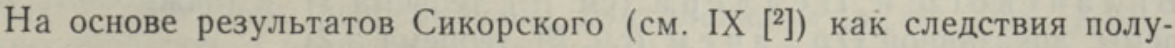
чаем две весьма интересные теоремы.

Те орем а 2.3. $X_{1}$-корегулярный метод $\mathfrak{A}$ не может быть сильнее $X_{1}$-конулевого метода $\mathfrak{B}$.

Т е о рем а 2.4. Если поля двух методов суммирования совпадают, то оба метода $X_{1}$-корегулярные или $X_{1}$-конулевые. 


\section{$\S 3$. Мощность методов}

Постараемся охарактеризовать методы суммирования через элементы $x \in X$, суммируемые этими методами. Для этого выберем такое подпространство $X_{2}$ пространства $X$, чтобы $X_{2}$ являлось $F$-пространством, $X_{1} \subset X_{2} \subset X$ и $X_{1} \neq X_{2} \neq X$ (теоретико-множественно). Будем рассматривать методы $\mathfrak{U}$ такие, что $X_{1} \subset \overline{\mathfrak{U}}$ (т. е. $X_{1}$-корегулярные или $X_{1}$-конулевые). Из определения $X_{1}$-конулевого метода вытекает.

Т ео рем а 3.1. $X_{1}$-конулевой метод суммирует элемент вне пространства $X_{2}$.

В самом деле, из определения $X_{1}$-конулевого метода вытекает, что каждый элемент $x \in X_{1}$ является точкой прикосновения множества $X_{0}$ в пространстве $\mathfrak{U}^{*}$. Так как это неверно в смысле топологии пространства $X_{2}$, то сразу получаем наше утверждение. На вопрос о том, когда $X_{1}$-корегулярный метод суммирует элемент вне пространства $X_{2}$, ответить несколько сложнее. Для ответа на него будем рассматривать две теоремы.

Т еорем а 3.2. Если в поле суммируемости $\mathfrak{U}^{*}$ метода $\mathfrak{A}$ каждый элемент $x \in X_{2} \cap \mathfrak{U}^{*}$ является точкой прикосновения множества $X_{1}$, то метод $\mathfrak{A}$ суммирует только элементы из $X_{1}$ или суммирует и элементы вне $X_{2}$.

Доказательство. Пусть $x^{0} \in X_{2} \cap \mathfrak{2}^{*}$ такой, что $x^{0} \in X_{1}$. В этом случае $x^{0}$ является точкой прикосновения множества $X_{1}$ в $\mathfrak{U}^{*}$. Так как это неверно в $X_{2}$, то должен существовать элемент $x^{1} \boldsymbol{\epsilon} \mathfrak{F}^{*}$ такой, что $x^{1} \bar{\epsilon} X_{2}$. Это и доказывает теорему.

Если пространство $X_{2}$ такое, что $\overline{\mathfrak{U}}=X_{2} \cap \mathfrak{U}^{*}$, то можно сформулировать теорему 3.2 несколько проще.

Т еорем а 3.3. Если $X_{1}$-корегулярный метод $\mathfrak{A}$, где $\overline{\mathfrak{A}}=X_{2} \cap \mathfrak{A}^{*}$, суммирует элемент вне пространства $X_{1}$, то он также суммирует элемент вне пространства $X_{2}$.

Доказательство. Надо убедиться в том, что каждый элемент $x \in \overline{\mathfrak{A}}$ является точкой прикосновения множества $X_{1}$. Но это вытекает из результата Сикорского (см. VIII $\left.\left[{ }^{2}\right]\right)$, так как метод $\mathfrak{Y}-X_{1}$-корегулярный.

Из теорем 3.2 и 3.3 видно, что $X_{1}$-корегулярный метод суммирует в первую очередь элементы вне пространства $X_{2}$, если он вообще суммирует элементы, не принадлежащие к пространству $X_{1}$. А в каком случае он суммирует элемент из пространства $X_{2}$ ? Частный ответ на этот вопрос дает нижеследующая теорема. В конкретных случаях из этой теоремы получаем необходимое и достаточное условие для того, чтобы метод $\mathfrak{U}=\left[X, \Re, v_{0}, N, n_{0},\{T 、\},\left\{f_{n}\right\}\right]$ суммировал элемент из пространства $X_{2}$. Эта теорема содержит как частный случай часть теоремы В ил ан ского и Целлер а (см. [11], теорема 1), а также часть теоремы, доказанной О р л и ч ем (см. [6], теорема 2.2).

Те о рем а 3.4. Если $\overline{\mathfrak{U}}=X_{2} \cap \mathfrak{A}^{*}$ и множество $X_{1}$ замкнуто в $\mathfrak{A}^{*}$, то метод $\mathfrak{Y}$ не суммирует элемента $x^{0} \in X_{2}$ такого, что $x^{0} \bar{\epsilon} X_{1}$.

Доказательство. Так как каждый элемент из $\overline{\mathfrak{Y}}$ является точкой прикосновения множества $X_{1}$ в $\mathfrak{U}^{*}$, то 


$$
X_{1} \subset \overline{\mathfrak{A}}=X_{2} \cap \mathfrak{A}^{*} \subset \bar{X}_{1},
$$

откдуа, ввиду замкнутости $X_{1}$, справедливо равенство $X_{2} \cap \mathfrak{U}^{*}=X_{1}$. Из этого и вытекает утверждение теоремы.

Интересно отметить, что в теореме 3.4 метод $\mathfrak{U}$ должен быть $X_{1}$-корегулярен. Действительно, при $X_{1}$-конулевом методе каждый элемент $x \in X_{1}$ является точкой прикосновения множества $X_{0}$ в $\mathfrak{P}^{*}$. Так как ввиду замкнутости $X_{1}$ топологии пространств $\mathfrak{U}^{*}$ и $X_{1}$ равносильны, то же самое обстоятельство должно иметь место и в $X_{1}$. Но это, очевидно, неверно.

\section{§ 4. Совместность методов}

Вопросы совместимости вышеуказанных методов излагал уже Сикорский [2]. Рассматривая два метода

$$
\mathfrak{N}=\left[X, \Re, v_{0}, N, n_{0},\left\{T_{v}\right\},\left\{f_{n}\right\}\right] \text { и } \mathfrak{B}=\left[X, \Re, v_{0}, M, m_{0},\left\{T_{v}\right\},\left\{g_{m}\right\}\right],
$$

он доказал следующие две теоремы.

Те орем а 4.1. Пусть $x \in \overline{\mathfrak{A}} u f(x)=\lim _{v \rightarrow v_{0}} f\left(x_{v}\right)$. Если метод $\mathfrak{B}$ не слабее метода $\mathfrak{A}$ и $f\left(x_{v}\right)=g\left(x_{v}\right)(v \in \Re)$, то $f(x)=g(x)$.

Т е о р ем а 4.2. Пусть существует элемент $x^{0} \in \overline{\mathfrak{A}}$ такой, что $f\left(x^{0}\right) \neq$ $\neq \lim _{v \rightarrow v_{0}} f\left(x_{v}^{0}\right)$. Если метод $\mathfrak{B}$ не слабее метода $\mathfrak{A} u \lim _{v \rightarrow v_{0}} f\left(x_{v}\right)=\lim _{v \rightarrow v_{0}} g\left(x_{v}\right)$, $\lim _{v \rightarrow v_{0}} f\left(x_{v} v_{v}^{0}\right)=\lim _{v \rightarrow v_{0}} g\left(x_{v}^{0}\right), f\left(x^{0}\right)=g\left(x^{0}\right)$, то $f(x)=g(x)$.

В настоящем параграфе мы обобщаем некоторые теоремы, хорошо известные для матричных методов, на случай метода Сикорского.

Т еорем а 4.3. Если $\mathfrak{A}=\left[X, \Re, v_{0}, N, n_{0},\left\{T_{v}\right\},\left\{f_{n}\right\}\right] X_{1}$-корегулярный u $\mathfrak{}=\left[X, \Re, v_{0}, K, \kappa_{0},\left\{T_{v}\right\},\left\{h_{k}\right\}\right] X_{1}$-конулевой методы, то не найдется совместного с $\mathfrak{C}$ метода $\mathfrak{B}=\left[X, \mathfrak{R}, v_{0}, \mathcal{M}, m_{0},\left\{T_{v}\right\}\left\{g_{m}\right\}\right]$, для которого $\mathfrak{B}^{*}=\mathfrak{A}^{*}$.

Доказательство. На основе теоремы 2.2 из условия $\mathfrak{A}^{*}=\mathfrak{B}^{*}$ вытекает, что метод $\mathfrak{B}$ является $X_{1}$-корегулярным.

С другой стороны, так как метод $\mathfrak{B}$ должен быть совместным с методом $\mathfrak{C}$, справедливо равенство $h(x)=g(x)$ при всех $x \in \mathfrak{B}^{*}$ (очевидно $\left.\mathfrak{B}^{*} \subset \mathrm{C}^{*}\right)$ и $h\left(x_{v}\right)=g\left(x_{v}\right)$ (множество $X^{\prime}$ принадлежит к полю всех методов 2 , $9 \mathcal{B}$ и (5). Из этого следует, что $g(x)=\lim _{v \rightarrow v_{0}} g\left(x_{v}\right)$ при всех $x \in X_{1}$ (т. е. $\mathfrak{B}$ является $X_{1}$-конулевым), так как $h(x)=\underset{v \rightarrow v_{0}}{\underset{v_{0}}{v_{0}}} h\left(x_{v}\right)$ прй всех $x \in X_{1}$.

Мы получили противоречие, что и доказывает теорему.

Учитывая то обстоятельство, что при $\mathfrak{B} * \supset \mathfrak{A}^{*} \mathfrak{B}$-сумма элемента $x$ является линейным непрерывным функционалом в $\mathfrak{I}^{*}$ (см. Х $\left.\left[{ }^{2}\right]\right)$, можно обобщить и некоторые результаты о совершенности матричных методов для числовых последовательностей на случай метода Сикорского. Мы называем метод 2 совершенным, если он совместен со всеми не более слабыми, чем он, методами, предполагая, что совместность имеет место на некотором множестве $E$. Рассматривая $X_{1}$-корегулярные и $X_{1}$-конулевые методы, выберем $E=X_{1}$. 
Очевидно, имеют место следующие теоремы:

Т е о ре м а 4.4. $X_{1}$-корегулярный метод $\mathfrak{A}$ совершенен тогда и только тогда, когда $X_{1}$ является основным множеством в $\mathfrak{2}^{*}$.

Теорема 4.4. обобщает теорему $6.4\left[^{8}\right]$.

Т е о ре м а 4.5. $X_{1}$-конулевой метод $\mathfrak{B}$ совершенен тогда и только тогда, когда $X_{0}$ является основным множеством в $\mathfrak{B}^{*}$.

\section{§ 5. ІІрименение к пространствам последовательностей}

В настоящем параграфе будем рассматривать методы суммирования

$$
\mathfrak{A}=\left[X, \mathfrak{H}_{,}, \boldsymbol{v}_{0}, N, n_{0},\left\{T_{v}\right\},\left\{f_{n}\right\}\right],
$$

которые определены преооразованием последовательностей. $\mathrm{K}$ этому классу принадлежат преобразования последовательности в последовательность, последовательности в ряд и последовательности в функцию. Последние образуют класс функциональных методов, рассмотренный В лод а рски м $\left.{ }^{12,13}\right]$. Во всех этих случаях возьмем $X=s$ (пространство всех числовых последовательностей), $X_{2}=m$ (пространство ограниченных последовательностей), $X_{1}=c$ (пространство сходяшихся последовательностей) и $X_{0}=c_{0}$ (пространство сходящихся к нулю последовательностей). Легко убедиться, что все выдвинутые треоования удовлетворены.

Возьмем

$$
T_{v}(x)=x_{v}=\left\{\xi_{0}, \xi_{1}, \ldots, \xi_{v}, 0,0, \ldots\right\}
$$

где $x=\left\{\xi_{k}\right\}$.

При матричных методах, определенных преобразованием последовательности в последовательность, $N$ и $\Re-$ множества всех натуральных чисел $\left(n_{0}=v_{0}=\infty\right)$. Функционалы $f_{n}$ определим равенством

$$
f_{n}\left(x_{v}\right)=\sum_{k=0}^{\nu} a_{n k} \xi_{k},
$$

где $\left(a_{n k}\right)$ - бесконечная матрица.

При преобразовании последовательности в ряд определим $N, \mathfrak{R}, n_{0}$ и $v_{0}$ как и в предыдущем случае, а

$$
f_{n}\left(x_{v}\right)=\sum_{i=0}^{n} \sum_{k=0}^{\nu} \alpha_{i k} \xi_{k}
$$

где $\left(\alpha_{i k}\right)$ - бесконечная матрица.

Рассматривая функциональные методы, определим $\Re$ и $v_{0}$ как и в предыдущих случаях. $N=\left[m_{0}, n_{0}\right)$ является интервалом, где $n_{0}$ конечно или бесконечно и

$$
f_{n}\left(x_{v}\right)=\sum_{k=0}^{\nu} a_{k}(n) \xi_{k},
$$

где $\left\{a_{k}(n)\right\}$ последовательность таких функций, определенных на $N$, что выполнено условие (c). 
Применяя соответствующие условия для сохранения сходимости (см. $[7,12])$, можно убедиться, что во всех этих случаях $\overline{\mathfrak{A}}=$ $=m \cap \mathfrak{U}^{*}$, если $\mathfrak{U}$ сохраняет сходимость. Из этого следует, что все результаты $\$ \$ 2-4$ применимы в рассмотренных здесь случаях.

Так как $c_{0}$ вместе с последовательностью $e=\{1,1, \ldots, 1, \ldots\}$ составляет основное множество в пространстве $c$, то метод $\mathfrak{A}$ является $c$-конулевым (или просто конулевым) тогда и только тогда, когда последовательности

$$
e^{r}=\underbrace{\{1,1, \ldots, 1}_{r \text { элементов }}, 0,0, \ldots\}
$$

слабо сходятся к $е$ в пространстве $\mathfrak{U}^{*}$. Из этого мы заключаем, что определение $c$-конулевого метода равносильно определению конулевого метода, которое Виланский дал для матричных методов в случае числовых последовательностей (см. $[10,4])$.

Сформулируем некоторые теоремы для функциональных методов.

Т е о рем а 5.1. Каждый функциональный конулевой метод суммирует неограниченную последовательность.

Т е орем а 5.2. Если корегулярный функциональный метод суммирует расходящуюся последовательность, то он суммирует и неограниченную последовательность.

Т еорем а 5.3. Функциональный метод $\mathfrak{U}$ не суммирует ограниченную расходящуюся последовательность тогда и только тогда, когда с замкнуто в $\mathfrak{2} *$

Т ео р е м 5.4. Функциональный корегулярный метод $\mathscr{U}$ совершенен тогда и только тогда, когда с составляет основное множество в $\mathfrak{U}^{*}$.

Теоремы $5.1,5.2$ и 5.4 непосредственно вытекают соответственно из теорем $3.1,3.3$ и 4.3. В случае теоремы 5.3 необходимость вытекает из теоремы 3.4 , а достаточность - из доказательства теоремы 3.1 М ейера-Kениг а и Целлер а (см. [5]).

Эти результаты известны для непрерывных функциональных методов, для которых они доказаны Влодарским [13] и Орличем [6]. Надо отметить, что определения непрерывных методов Влодарского и Орлича немного отличаются одно от другого, но оба они такие, что условие (с) выполнено.

\section{§ 6. Применение к пространствам рядов}

Рассмотрим применения вышеприведенных теорем для счулая, когда $X_{0}, X_{1}, X_{2}$ и $X-$ «пространства рядов», т. е. такие пространства последовательностей, где элементами последовательностей являются члены некоторых рядов. При помощи различных функционалов можем получить методы, определенные преобразованиями ряда в ряд и ряда в функцию.

Во всех этих случаях примем за пространства $X, X_{2}, X_{1}$ и $X_{0}$ соответственно пространство всех числовых рядов $-s_{r}$, пространство всех рядов с ограниченнымн частными суммами $-m_{r}$, пространство всех сходящихся рядов $c_{r}$ и пространство всех сходящихся к нулю рядов - $n_{r}$. Все они оказываются $F$-пространствами, если квазинормы точки $x=\left\{\xi_{k}\right\}$ определить следующим образом: 
в пространстве $s_{r}: \quad\left|\xi_{k}\right| \quad(k=0,1, \ldots)$;

в пространствах $m_{r}, c_{r}$ и $n_{r}: \sup _{0 \leqslant n<\infty}\left|\sum_{k=0}^{n} \xi_{k}\right|$.

Для применения общей теории, развитой в $\$ 2-4$, надо определить оператор $T_{\nu}$ таким образом, чтобы $T_{\nu}(x)=x_{v} \in n_{r}$. Определим

$$
T_{\nu}(x)=\sum_{k=0}^{\nu} \xi_{k}\left(e_{k}-e_{v+1}\right),
$$

где

$$
e_{k}=\{\underbrace{0, \ldots, 0}_{\kappa \text { нулей }}, 1,0, \ldots\} \quad(k=0,1, \ldots) .
$$

Из этого определения видно, что

$$
x_{v} \in n_{r} \subset s_{r},
$$

а также, что выполнены условия (a) и (b) (см. §1).

Рассматривая преобразование ряда в последовательность, определим

$$
f_{n}\left(x_{\nu}\right)=\sum_{k=0}^{\nu}\left(\alpha_{n k}-\alpha_{n, v+1}\right) \xi_{k},
$$

и проверим, когда выполнены условия $1^{0}-3^{0}$ (см. $\S 1$ ), если преобразование

$$
\eta_{n}=\sum_{k=0}^{\infty} a_{n k} \xi_{k} \quad(n=0,1, \ldots)
$$

сохраняет сходимость.

Метод $\mathfrak{U}=\left(\alpha_{n k}\right)$ преобразования ряда в последовательность сохраняет сходимость тогда и только тогда, когда

1) существует $\lim _{n \rightarrow \infty} \alpha_{l i k}=\alpha_{k} \quad(k=0,1, \ldots)$,

2) $\sum_{k=0}^{\infty}\left|\alpha_{n k}-\alpha_{n, k+1}\right| \leqslant M \quad(n=0,1, \ldots) \quad$ (см. $\left.\left[{ }^{1}\right]\right)$.

Легко проверить, что из условий 1) и 2) вытекает, что $1^{0}-3^{0}$ выполнены, если $x \in m_{r} \cap \mathfrak{Y}^{*}$, т. е. $\overline{\mathfrak{H}}=m_{r} \cap \mathfrak{U}^{*}$. Последнее обстоятельство позволяет применить общую теорию и рассматриваемому случаю.

\section{§ 7. Применение к интегральным методам}

Рассмотрим, наконец, интегральный метод суммирования функций, определенный преобразованием

$$
y(n)=\int_{0}^{\infty} K(n, t) x(t) d t
$$


Этот метод является частным случаем общего метода суммирования Сикорского (см. [ㄹ]).

Пусть $X=L$ (пространство всех ф̈ункций, определенных на $[0, \infty)$ и интегрируемых в смысле Лебега на каждом конечном сегменте $[0, t])$. Пространство $L$ является $F$-пространством с квазинормами

$$
p_{r}(x)=\int_{0}^{r}|x(t)| d t \quad(r=1,2, \ldots) .
$$

Пусть $N=\Re=[0, \infty), n_{0}=v_{0}=\infty$ и

$$
T_{v}(x)=x_{v}=\left\{\begin{array}{c}
x(t), t \leqslant v, \\
0, t>v .
\end{array}\right.
$$

Функция $x_{v}$ называется отрезком функции $x=x(t)$. Из общей теории получим интегральный метод, если

$$
f_{n}\left(x_{v}\right)=\int_{0}^{v} K(n, t) x(t) d t,
$$

где функция $K(n, t)$ такая, что выполнены условия (c) и $(\mathrm{d})$.

Для применения общих результатов надо определить $F$-пространства $X_{0}, X_{1}$ и $X_{2}$ таким образом, чтобы

$$
X_{0} \subset X_{1} \subset X_{2} \subset X .
$$

Возьмем $X_{2}=M, X_{1}=C$ и $X_{0}=C_{0}$, где

$M$ - пространство всех ограниченных на $[0, \infty)$ функций $x(t)$ с нормой

(2)

$$
\|x\|=\sup _{0 \leqslant t<\infty}|x(t)| .
$$

$C$ - пространство всех функций $x(t) \in M$, для которых существует $\lim x(t)$ с нормой (2) (пространство сходящихся функций); $\lim _{t \rightarrow \infty} x(t$

$C_{0}$ - пространство всех функций $x(t) \in C$, для которых $\lim _{t \rightarrow \infty} x(t)=0$ с нормой (1).

Учитывая условия, необходимые и достаточные для того, чтобы интегральный метод $\mathfrak{A}$ сохранял сходимость (см. [9]), можно убедиться, что $\overline{\mathfrak{A}}=M \cap \mathfrak{U}^{*}$. Из общей теории (\$\$ 2-4) получим, например, следующие теоремы:

т е о рем а 7.1. Если С-корегулярный интегральный метод суммирует расходящуюся функцию, то он также суммирует неограниченную функциию.

Т е о р е м 7 7.2. Ннтегральный $C$-корегулярный метод является совершенным тогда и только тогда, когда С есть основное множество в поле суммируемости.

Аналогично доказательству одной теоремы Мейера-Кенига и Целлера (см. [5], теорема 3.1) можно доказать и следующую лемму:

Л е м м а 7.3. Пусть $R$ является некоторым $F$-пространством функиций $x(t) \quad(0 \leqslant t<\infty)$, в котором имеет место сходимость по координатам. Если ограниченные отрезки одни или вместе с постоянными функциями 
составляют основное множество в $R$, то либо при каждом $x(t) \in R$ существует $\lim x(t)$, либо $R$ содержит такюе ограниченную расходящуюся $t \rightarrow \infty$

функцию.

С помощью этой леммы из общей теории получим следующие тео: ремы:

Т еорем а 7.4. Интегральный $C$-корегулярный метод не суммирует ограниченную расходящуюся функцию тогда и только тогда, когда С замкнуто в поле суммируемости.

Те о рем а 7.5. Каждый $C$-конулевой интегральный метод суммирует как неограниченную, так и ограниченную расходящуюся функцию.

Т е о рем а 7.6. Каждый совершенный $C$-корегулярный интегральный метод суммирует как неограниченную, так и ограниченную расходящуюся функцию, если он вообще суммирует функции, кроме сходямихся.

\title{
Л ИТ Е Р А Т У Р А
}

1. Г. Ф. К а н гр о, О множителях суммируемости, Уч. зап. Тартуск. ун-та, 37, 1955.

2. Р. Сикорский, Некоторые замечания о теории суммируемости Мазура-Орлича, Бюл. Польской АН, отд. 3, 3, 1955.

3. Г. Х арди, Расходящиеся рялды, 1951.

4. Э. И. Ю р и м я э, Некоторые вопросы обобщенных матричных методов суммирования. Корегулярные и конулевые методы, Изв. АН Эст. ССР. Сер. техн. и физ,-мат. наук, т. VIII, № 2, 1959.

5. W. Me y e r-König, K. Zeller, Lückenumkehrsätze und Lückenperfektheit, Math. Z., 66, 1956.

6. W. Orlicz, On the Summability of Bounded Sequences by Continuous Methods, Bull. Acad. polon. sci., Cl. 3, 6, 1958.

7. J. Schur, Uber lineare Transformationen in der Theorie der unendlichen Reihen, J. reine und angew. Math., 151, 1921.

8. K. Z e 11 e r, Allgemeine Eigenschaften von Limitierungsverfahren, Math. Z., 53, 1951.

9. K. Zelle r, Uber Stetigkeit von Integraltransformationen, Math. Z., 55, 1952.

10. A. Wilansky, An Application of Banach Linear Functionals to Summability, Trans. Amer. Math. Soc., 67, 1949.

11. A. Wilansky and K. Zeller, Summation of Bounded Divergent Sequences, Topological Methods, Trans. Amer. Math. Soc., 78, 1955.

12. L. Wlodarski, Sur les méthodes continues de limitation (I), Studia math., 14, 1954.

13. L. Wlodarski, Sur les méthodes continues de limitation (II), Studia math., 14, 1954.

Тартуский государственный университет

Поступила в редакцию

11. XI 1959.

\section{KOONDUVUST SÄILITAVAD SUMMEERIMISMENETLUSED}

\author{
E. Jürimäe, \\ füüsikalis-matemaatiliste teaduste kandidaat
}

Resümee

Käesolevas töös vaadeldakse Sikorski $\left.{ }^{2}\right]$ poolt defineeritud summeerimismenetlusi. Nende jaoks üldistatakse mõisted «koonduvust säilitav», «konull-» ja «koregulaarne» menetlus (§ 2). See vôimaldab antud menetluste jaoks üle kanda mõned arvjadade puhul tuntud omadused, näiteks: tōkestamata elemendi olemasolu menetluse summeerimisväljas, tõkestatud elementide summeerimise, menetluste kooskôla ja perfektsuse. Üldisi teoreeme rakendatakse maatriksmenetluste (jada-jada ja rida-jada kujul) puhul, samuti ka pidevate menetluste ja integraalmenetluste puhul. 


\section{KONVERGENZTREUE SUMMIERUNGSVERFAHREN}

\section{E. Jürimäe}

\section{Zusammenfassung}

In vorliegender Arbeit werden die von Sikorski $\left[{ }^{2}\right]$ definierten Summierungsverfahren betrachtet. Für diese Verfahren werden die Begriffe der «konvergenztreuen», «co-null» und «co-regulare» Transformationen verallgemeinert $(\$ 2)$. Die Zugrundelegung dieser Definitionen ermöglicht einige für Zahlenfolgen schon bekannte Eigenschaften auch auf die obenerwähnten Summierungsverfahren zu übertragen, z. B. die Existenz unbeschränkter Elemente im Wirkfeld, die Summierung beschränkter divergenter Elemente, die Verträglichkeit und Perfektheit der Verfahren. Die allgemeinen Sätze werden auf die Matrixverfahren (in Folge-Folge- und Reihe-Folge-Form), ausserdem auch auf die stetigen Verfahren und auf die Integralverfahren angewandt. 